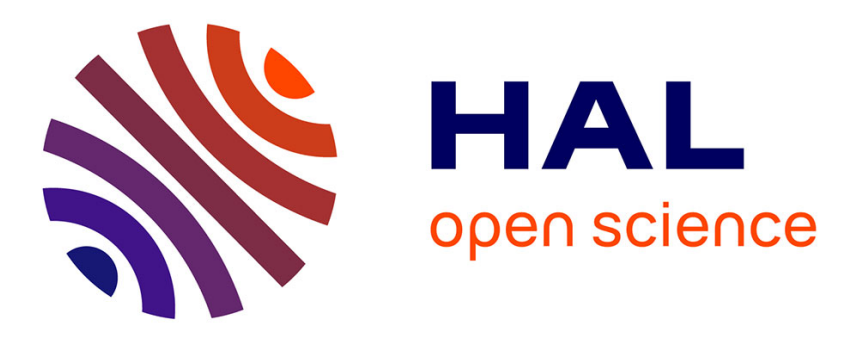

\title{
Polysilicon Nanowires for chemical sensing applications
}

Emmanuel Jacques, Liang Ni, Anne-Claire Salaün, Régis Rogel, Laurent

Pichon

\section{To cite this version:}

Emmanuel Jacques, Liang Ni, Anne-Claire Salaün, Régis Rogel, Laurent Pichon. Polysilicon Nanowires for chemical sensing applications. MRS, Spring Meeting, Apr 2012, San Francisco, United States. pp.133. hal-00795800

\section{HAL Id: hal-00795800 https://hal.science/hal-00795800}

Submitted on 1 Mar 2013

HAL is a multi-disciplinary open access archive for the deposit and dissemination of scientific research documents, whether they are published or not. The documents may come from teaching and research institutions in France or abroad, or from public or private research centers.
L'archive ouverte pluridisciplinaire HAL, est destinée au dépôt et à la diffusion de documents scientifiques de niveau recherche, publiés ou non, émanant des établissements d'enseignement et de recherche français ou étrangers, des laboratoires publics ou privés. 


\title{
Polysilicon Nanowires for chemical sensing applications
}

\author{
E. Jacques, L. Ni, A. C. Salaün, R. Rogel, L. Pichon \\ Institut d'Electronique et de Télécommunications de Rennes, UMR 6074, Campus de Beaulieu, \\ bâtiment 11 B, 263 avenue du Général Leclerc, \\ 35042 Rennes cedex, France
}

\begin{abstract}
Polycrystalline silicon nanowires are synthesized using a classical fabrication method commonly used in microelectronic industry: the sidewall spacer formation technique. Assets of this technological process rest on low cost lithographic tools use, classical silicon planar technology compatibility and the possibility to get by direct patterning numerous parallel nanowires with precise location on the substrate. Grounded and suspended polycrystalline silicon nanowires with a curvature radius as low as $150 \mathrm{~nm}$ are integrated into resistors and used as gas (ammonia) sensors. Results show potential use of these nanowires for charged chemical species detection with an increase of the sensitivity with the increase of SiNWs exchange surface with the environment.
\end{abstract}

\section{INTRODUCTION}

Semiconducting nanowires are currently attracting much attention as promising components for future nanoelectronic devices such as nanowire field effect transistors [1], photonic and optoelectronic devices [2], and as chemical or biological sensors [3-5]. As their surface can be sensitive to charged species combined with their high surface to volume ratio, 
silicon nanowires (SiNWs) are the subject of intense research activities for high sensitivity chemical sensor fabrication. The first application of SiNWs in biological and chemical molecule sensor was reported by Lieber's group in 2001 [3]. In addition, because SiNWs synthesis is compatible to the established Si technology, enormous research efforts to design and develop new generation of high performance biological and chemical sensors by incorporating the SiNWs as the functional sensitive units are performed. Indeed, SiNWs based sensor integration will allow a lower manufacturing cost, in addition to the advantageous electronic features of embedded detection and signal processing in silicon technology. The intrinsic reliability of the well-known semiconductor CMOS (Complementary Metal Oxide Semiconductor) process also guarantees a reproducible and reliable diagnosis.

SiNWs can be prepared by one of two approaches, "top-down" and "bottom up". In a bottom up strategy the individual base elements (atoms, molecules...) of the system are linked together to form larger subsystems. Synthesis methods most developed are layer-by-layer self assembly [6], Vapor Liquid Solid (VLS) and Solid Liquid Solid (SLS) growth techniques [7,8], and use of matrix template [9]. The main drawbacks of these synthesis methods for a $3 \mathrm{D}$ integration are the difficulty in control of size and positioning of the nanowires. In this case, nanowires need to be selectively collected and manipulated to be assembled in a planar layout. The "top down" approach starts from bulk materials and scales down the patterned areas. In this way, several advanced nanopatterning techniques were developed such as e-beam [10], atomic force microscopy (AFM) [11,12], deep UV [13] and nanoimprint lithographies [14,15], to obtain SiNWs. The main drawbacks of these advanced lithographic tools with nanometer size resolution rest on the high cost generated, and more generally the low throughput capability unsuitable with mass production. 
In this study, we present a direct patterning technique to get SiNWs planar arrays by conventional optical lithography, compatible with the existing planar CMOS silicon technology. Such SiNWs are promising active building blocks for gas (ammonia) sensors.

\section{EXPERIMENTAL DETAILS}

\section{Spacer polycristalline SiNWs fabrication}

The key fabrication steps of the polycrystalline silicon NWS are illustrated in figure 1. At first, a dielectric film A is deposited and patterned into islands by conventional UV lithography. Then, a polycrystalline silicon layer is deposited by low pressure chemical vapor deposition (LPCVD) technique and plasma etched by anisotropic reactive ion etching (RIE). Accurate control of the polycrystalline silicon layer RIE rate leads to the formation of nanometric size sidewall spacers that can be used as nanowires. The feasibility of these polyscrystalline silicon NWs with curvature radius as low as $50 \mathrm{~nm}$ was previously demonstrated [16]. This method allows the fabrication of parallel SiNWs network

\section{SiNWs based devices fabrication}

Two types of devices used as gas (ammonia) sensors are fabricated as illustrated schematically in figure 2: grounded or suspended SiNWs based resistors. For each type of resistor, the fabrication of spacer polycrystalline SiNWs follows step described in the figure 1 . 
Next, a highly in-situ doped polycrystalline silicon layer is deposited by LPCVD technique at $550^{\circ} \mathrm{C}$ in the amorphous state and crystallized by thermal annealing at $600^{\circ} \mathrm{C}$ and patterned by

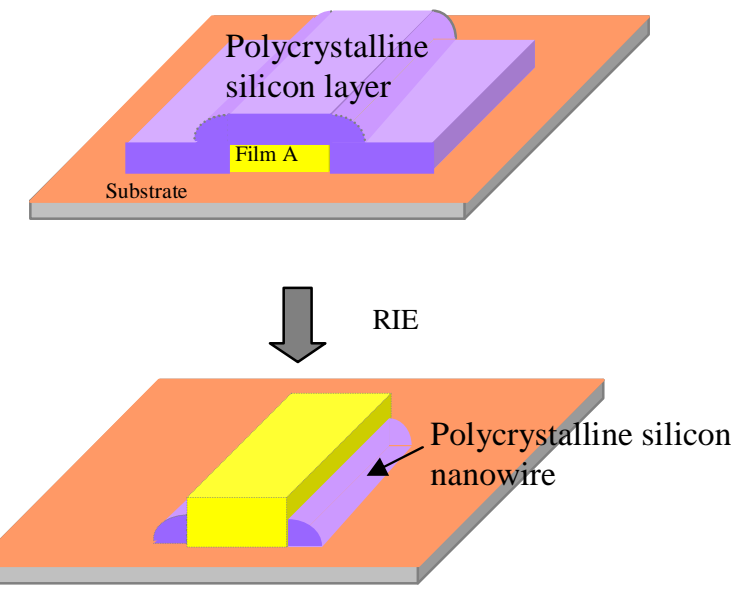

Figure 1. Fabrication of polycrystalline silicon NWs by the sidewall spacer formation technique

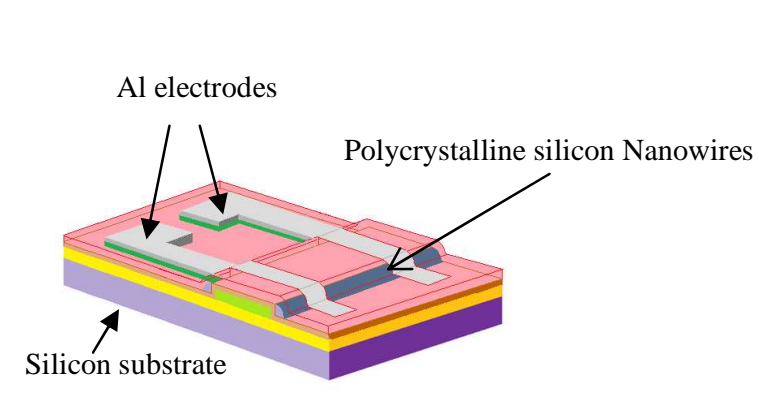

grounded SiNWs based resistor

(a)

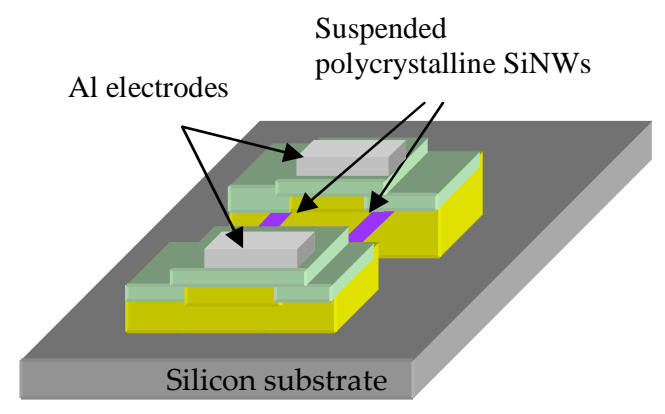

suspended SiNWs based resistor

(b)

Figure 2. Grounded (a), suspended (b) SiNWs based resistors

RIE to form electrical contacts. The last step includes electrodes patterning using thermally evaporated aluminium (fig. 2 (a). For the suspended SiNWs based devices wet etching of the 
$\mathrm{SiO}_{2}$ sacrificial layer (film A in fig. 1) allows the suspended SiNWs formation anchored between two highly in-situ doped polycrystalline silicon electrical contacts (fig. 2 (b))

\section{Electrical measurements}

Performances of SiNWs based resistors are characterized measuring static electrical characteristics I-V using a probing tester and Agilent Technologies B1500A semiconductor device analyzer. The resistance $(\mathrm{R}=\mathrm{V} / \mathrm{I})$ is deduced from the slope of the linear $\mathrm{I}-\mathrm{V}$ curves for each resistor. R-values are then reported as function of time for devices under exposure to gas. Prior to measurements, devices are submitted to highly diluted hydrofluoric acid (2\%) aqueous solution to remove the native oxide on the SiNWs surface in order to promote chemical species adsorption. Then, SiNWs based devices are put into a vacuum chamber. The electrical measurements are carried out at room temperature and the pressure is monitored at 500mbar regardless of the gas flow (ammonia) injection.

At first, a flow of nitrogen is injected continuously into the chamber and the current measurement as a function of time is started. After the current stabilizes, a flow of ammonia is injected with the chosen ratio of $\mathrm{NH}_{3} / \mathrm{N}_{2}$. After a certain period of time exposure, the gases injection is cut off. Then, the mixture gas of $\mathrm{NH}_{3} / \mathrm{N}_{2}$ is evacuated to get back to the environment at the initial state. During the whole period of measurement, the mixture of gas $\mathrm{NH}_{3} / \mathrm{N}_{2}$ is exchanged all the time whereas the concentration of the gases is constant. This means that the dynamic protocol is suitable to detect a certain concentration of gases flowing through a fixed space. 
The potential use of SiNWs as sensitive units to ammonia detection is checked by measuring the detector response, $S_{\mathrm{g}}$, defined as:

$$
S_{g}=\frac{R_{g}-R}{R_{g}}=\frac{I-I_{g}}{I}
$$

where $R(I)$ and $R_{g}\left(I_{g}\right)$ are the resistance (current) values for devices under nitrogen and reactive ambient respectively.

\section{DISCUSSION}

Figure 3 shows SEM pictures of grounded and suspended polycrystalline silicon nanowires with a 100nm curvature radius. Thanks to an accurate control of the etching rate of the polysilicon waste, the sidewall spacer formation method allows fabrication of SiNWs array network. These results highlight the feasibility of this technological step with a precise control on size, orientation and good mechanical robustness for the suspended polycrystalline SiNWs.

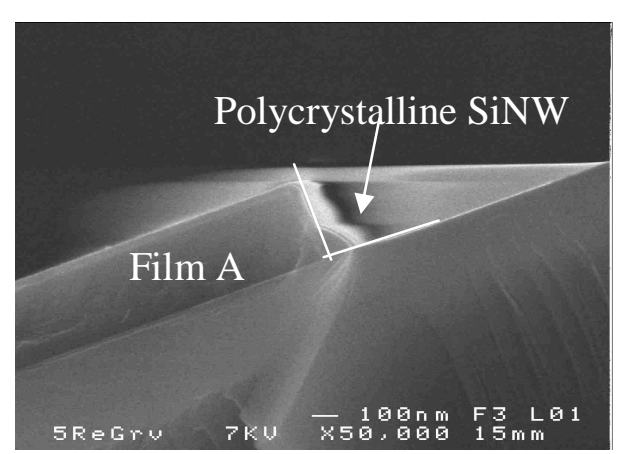

(a)

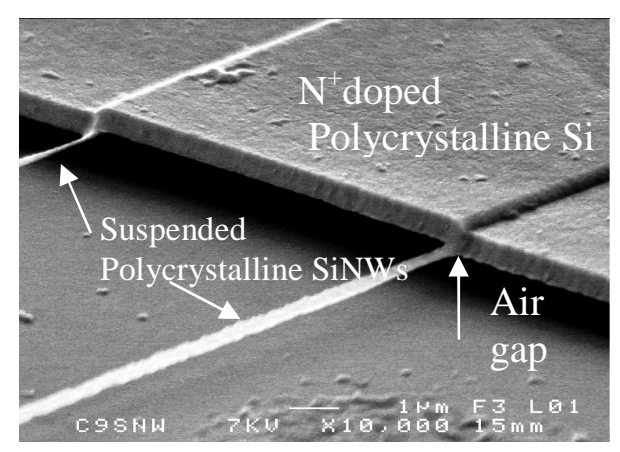

(b)

Figure 3. SEM pictures of: (a) grounded (after [16]), (b) suspended spacer polycrystalline SiNWs. 
Such nanowires are then integrated into the fabrication of electrical resistors used as gas (ammonia) sensors. The feasibility of the electrical resistors based on the two types of polycrystalline silicon nanowires is first demonstrated by I-V curves plotted in the figure 4 . The lower current level for the suspended SiNWs based resistors could be explained by a possible

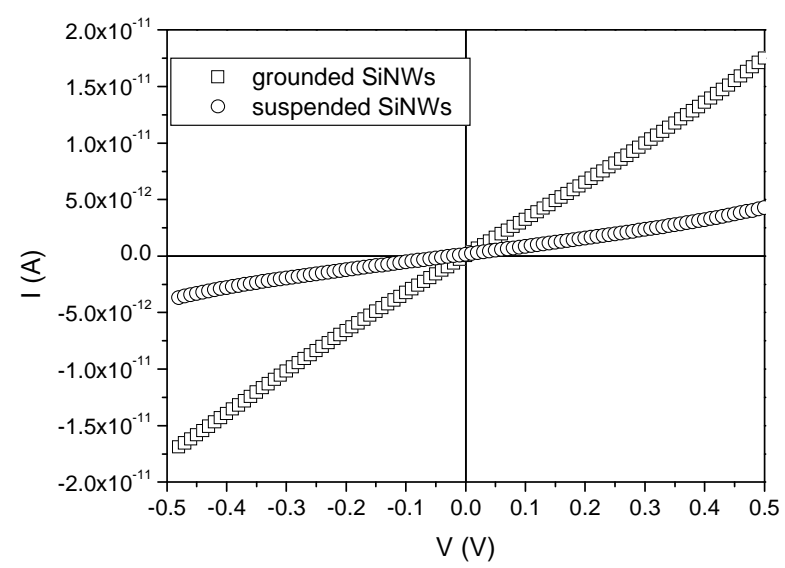

Figure 4. I-V characteristics of grounded and suspended polycrystalline SiNWs based resistors. SiNWs radius curvature: $150 \mathrm{~nm}$.

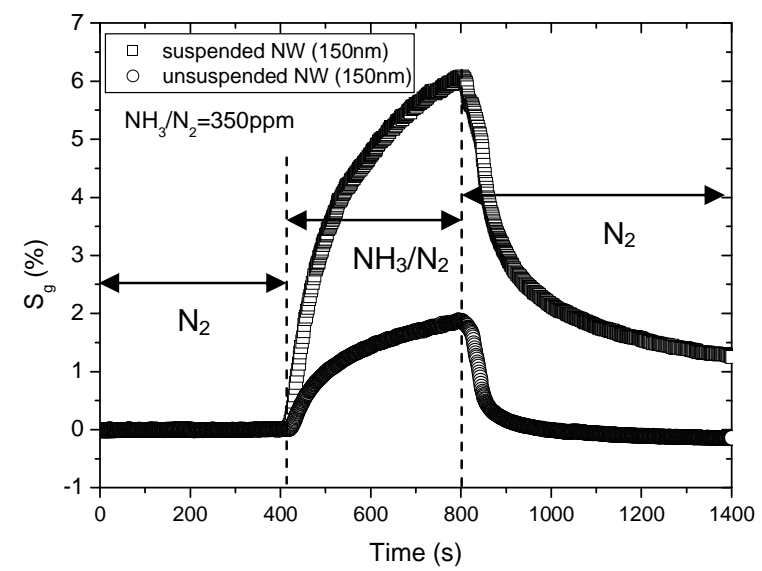

Figure 5. Variations of the detector response versus time under controlled ammonia (350ppm) exposure test. The pressure adjusted in the chamber is 500mbar, and measurements are carried out at room temperature, for a bias voltage of $0.5 \mathrm{~V}$. 
etching excess of the SiNW during the 15 min duration $\mathrm{SiO}_{2}$ sacrificial layer wet etching, using a buffer hydrofluoric acid aqueous solution. Such etching excess may reduce the SiNW radius curvature and thus leads to the increase of the resistance of the suspended SiNW.

The sensitivity of the SiNWs to ammonia species is reported in the figure 5 for the measurement of detection response, $\mathrm{S}_{\mathrm{g}}$, with a bias voltage of $0.5 \mathrm{~V}$ at room temperature. Upon exposure to ammonia electrical resistance decreases because ammonia species act as donor of electrons (reducing agents) at the SiNWs surface [17]. As the ammonia gas molecules adsorption occurs at the SiNWs surface, electrons are transferred to the SiNWs crystal core. Such interactions induce significant changes in the carriers transport along the nanowires and thus for SiNWs electrical resistance in different possible cumulative ways. First, as the SiNWs conductance can be modulated by an applied voltage [17, 18], positively charged gas molecules (electron donors) bonded on SiNWs surface can modulate their conductance by changing the volume of the conductive layer. In this case, ammonia may act as chemical gates. In other words, it means that the Fermi level of the $\mathrm{Si}$ nanowires is shifted reducing the sample electrical resistance.

The higher chemical response for the suspended based resistors is related to the higher exchange surface of the SiNWs with the environment. In this case, for a constant gas mixture, a higher exchange SiNW surface allows a higher number of bonded ammonia molecules on the SiNWs surface leading to a higher electrons concentration taking part to carriers transport into the SiNWs. 


\section{CONCLUSIONS}

Based on this nanospacer method, 2D polycrystalline SiNW arrays can be fabricated, at low-temperature $\left(<600^{\circ} \mathrm{C}\right)$, by direct patterning technique using classical lithographic tools, compatible with established silicon technology. Results show that such SiNWs can be used as potential sensitive units for integrated gas (ammonia) sensors applications operating at room temperature. Such SiNWs based sensor integration will allow a lower manufacturing cost, in addition to the advantageous electronic features of embedded detection and signal processing in CMOS silicon technology.

In addition, thanks to silicon surface functionalization possibilities, such results offer a great potential for further developments of integrated SiNWs based (bio)chemical sensors .

\section{ACKNOWLEDGMENTS}

This work was supported by the Agence Nationale de la Recherche (France) with the Project Sena (ANR-09JCJC-0072-01).

\section{REFERENCES}

1. J. Goldberger, A. I. Hochbaum, R. Fan, P Yang, Nanoletters 6(5), 973 (2006)

2. C. Yang, C. J. Barrelet, F. Capasso, C.M. Lieber, Nanoletters 6(12), 2929 (2006)

3. Y. Cui, Q. Wei, H. K. Park, C. M. Lieber, Science, 293(5533), 1289 (2001) 
4. L.M. Lechuga J. Tamayo, M. Álvarez, L.G. Carrascosa, A. Yufera, R. Doldán, E. Peralías, A. Rueda, J.A. Plaza, K. Zinoviev, C Domínguez, A. Zaballos, M. Moreno, C. Martínez-A, D. Wenn, N. Harris, C. Bringer, V. Bardinal, T. Camps, C. Vergnenègre, C. Fontaine, V. Diaz, A. Bernad, Sensors and Actuators B 118, 2 (2006)

5. Z. Li, Y. Chen, X. Li, T.I. Kamins, K. Nauka, R. S. Williams, Nanoletters 4(2), 245 (2004)

6. Liu Y, Cui T, J Nanosci. Nanotechnol 6(4), 1019 (2006)

7. P. Cheyssac, M. Sacilotti, and G. Patriarche, J. Appl. Phys. 100, 044315 (2006)

8. L.Yu. P Roca i Cabarrocas, Physical Review B 81, 085323 (2010)

9. I. Michelakaki, A. G. Nassiopoulou, E. Stavrinidou, K. Breza, N. Frangis, Nanosc. Res. Lett. 6, 414 (2011)

10. H. Xiang-Lei, L. Guilhem, F. Pier-Francesco, D. Emmanuel, Microelectr. Eng., 88 (8), $2622(2011)$

11. I. Ionica, L. Montes, S. Ferraton, J. Zimmermann, L. Saminadayar,V. Bouchiat, Sol. St. Electr. 49, 1497 (2005)

12. L Pichon, R Rogel, F Demami, Semicond. Sci. Technol. 25, 065001 (2010)

13. L. Yang, D. H. Lee, Y. H. Chen, C. Y. Chang, S. D. Liu, C. C. Huang, VLSI Symp. Tech. Dig, p196 (2004)

14. S.Y. Chou, R. Krauss, P. J. Renstrom, Science, 85, 272 (1996)

15. A. Talin, L. Hunter, F. Léonard,B. Rokad, Appl. Phys. Lett. 89, 153102 (2006)

16. F. Demami, L. Pichon, R. Rogel, A. C. Salaun, Mat. Sc. Eng. 6012014 (2009)

17. A. A. Talin, L. L. Hunter, F. Leonard, B. Rokad, Appl. Phys. Lett, 89, 153102 (2006)

18. J.Y. Yu, S.W. Chung, J.R. Heath, Jour. of Phys. Chem. B 104, 11864 (2000) 
